\title{
Manipulation of intestinal dysbiosis by a bacterial mixture ameliorates loperamide- induced constipation in rats
}

\author{
Y. Deng ${ }^{1 \#}$, M. Li ${ }^{1 \#}$, L. Mei ${ }^{2}$, L.M. Cong1, Y. Liu', B.B. Zhang ${ }^{3}$, C.Y. He ${ }^{1}$, P.Y. Zheng ${ }^{2 *}$ and J.L. Yuan $^{1^{*}}$ \\ ${ }^{1}$ Department of Microecology, College of Basic Medical Science, Dalian Medical University, Dalian, China P.R.; ${ }^{2}$ Department \\ of Gastroenterology, the Second Affiliated Hospital of Zhengzhou University, Zhengzhou, China P.R.; ${ }^{3}$ Laboratory of Pathogenic \\ Biology, College of Basic Medical Science, Dalian Medical University, Dalian, China P.R.; jieli_yuan_dmu@163.com; \\ medp7123@126.com; \# these authors contributed equally to this work
}

Received: 23 May 2017 / Accepted: 7 November 2017

(c) 2018 Wageningen Academic Publishers

OPEN ACCESS CC)

\begin{abstract}
Constipation has a significant influence on quality of life. Patients with constipation have slow waves in their gastrointestinal smooth muscles and less faecal water contents, which are closely associated with down-regulation of the interstitial cells of Cajal (ICC) in the gastrointestinal muscles and the aquaporin protein AQP3 expressed in colon epithelial cells. Recent studies supported that patients with constipation have altered intestinal microbial structures compared with healthy controls. Intestinal dysbiosis might be one possible pathophysiological mechanism causing constipation. Bacterial strains, such as Lactobacillus spp., have shown many beneficial effects on the amelioration of constipation. However, few studies reported the structural changes of intestinal microbiota post-intervention of probiotics. In this study, a bacterial mixture was administrated to rats with loperamide-induced constipation. Effects of the bacterial mixture on small intestine transit (SIT), faecal water content, and the intestinal microbiome in rats were evaluated. Meanwhile, we investigated several factors involved in signalling pathways that regulate function of ICC and expression of AQP3 to discuss the possible underlying molecular mechanisms. Intervention of the bacterial mixture improved SIT and faecal water content in constipated rats. The up-regulation of C-kit/ SP signalling pathways in ICC and AQP3 significantly contributed to improvements. These changes were closely associated with the manipulation of intestinal dysbiosis in constipated rats. Furthermore, our results revealed the important role of intestinal microbiota in affecting gut motility through regulation of serotonin biosynthesis. This monoamine neurotransmitter, secreted from enterochromaffin cells, up-regulated both substance $\mathrm{P} /$ neurokinin 1 receptors pathway of ICC and the expression of AQP3 in intestinal epithelial cells. Our study suggested that the disrupted microbiome in patients could be a potential therapeutic target for the improvement of constipation.
\end{abstract}

Keywords: constipation, dysbiosis, probiotics, ICC, serotonin

\section{Introduction}

Functional constipation (FC) is a symptom of many disorders, such as prolonged faecal stasis in the colon, difficulties during defecation, and infrequent bowel movements. According to epidemiological investigations, the prevalence of constipation ranged from 2 to $28 \%$ in adults and had a positive correlation with aging. With the accelerated pace of modern society, as well as changes in people's diet, the incidence of chronic constipation showed a gradual upward trend. Constipation has a significant influence on quality of life and might be related to mental illness (Suares and Ford, 2011).

Compared with healthy controls, patients with constipation had slower waves in their gastrointestinal (GI) smooth muscles. Slow waves are rhythmic oscillations in the membrane potential and are initiated by the interstitial cells of Cajal (ICC) in the GI muscles (Huizinga et al., 1995). The ICC are closely associated with varicosities of the enteric nerves, which mediate inhibitory and excitatory nerve signals to smooth muscles (Koh et al., 
1998). Neurotransmitter substance P (SP) was found to play important roles for excitation of ICC (Sanders, 1996). SP exerts a potent contractile effect on GI smooth muscle through neurokinin (NK) receptors (especially $\mathrm{NK}_{1}$ ) that modulated $\mathrm{Ca}^{2+}$ and $\mathrm{Cl}^{-}$channels on ICC (Daniel, 2001; Jun et al., 2004; Watson and Downes, 1983). Reduced faecal water content is also usually found in patients with FC and closely related to the disturbance of water transport system in patient's colon. Aquaporins (AQPs) play important roles in the water transport system in the human body, changing of the expression levels of AQP3 in colon epithelial cells causes severe constipation (King et al., 2004; Zhu et al., 2017). Activation of protein kinase A (PKA) in HT-29 cells increased the expression levels of AQP3, resulting in increased faecal water content. This effect was related to the increase in the intracellular $\mathrm{Mg}^{2+}$ concentration that triggered cAMP response element binding protein (CREB) phosphorylation after PKA activation (Ikarashi et al., 2011). In addition, serotonin (5-HT) secreted from enterochromaffin cells (ECs) up-regulated both SP/NK1 receptors pathway in colonic nerve fibres (Utsumi et al., 2016) and the expression of AQP3 in intestinal epithelial cells (Kon et al., 2015). These results suggest a key regulatory role of 5-HT in regulating GI motility.

Biosynthesis of 5-HT by ECs is regulated by indigenous bacteria in the human gut, suggesting a close interaction between the gut microbiome and GI motility (Yano et al., 2015). Actually, in recent years, intestinal dysbiosis was suggested as one of the possible pathophysiological mechanisms of FC. Khalif et al. (2005) reported that the gut microbial structure of constipated adults differed from that of healthy adults. The decreased abundance in bifidobacteria exhibited as the major difference of the constipated microbiome (Zoppi et al., 1998). Groups of Clostridia were found significantly increased in the gut of constipated children and the Clostridium species isolated from constipated children were different from healthy children (Khalif et al., 2005). A recent study (Zhu et al., 2014) demonstrated that Prevotella was significantly decreased, while several representative genera of Firmicutes were increased in constipated subjects. These studies suggested that manipulation of intestinal dysbiosis contributed to the improvement of FC. Probiotics, such as Bifidobacterium and Lactobacillus, could affect the intestinal peristalsis through fermenting food, producing gas and short chain fatty acids. Probiotics were also effective in manipulating disrupted intestinal microbial structures (Jeong et al., 2017; Yu et al., 2017). However, to date, few reports have studied the structural changes of intestinal microbiota post-intervention of probiotics. In addition, whether disrupted intestinal equilibrium by probiotics can affect the regulatory pathways of ICC and the expression of AQP3 remains unclear.
In this study, a bacterial mixture (Bifidobacterium breve DM8310, Lactobacillus acidophilus DM8302, and Lactobacillus casei DM8121) was administered to rats with loperamide-induced constipation. Effects of the bacterial mixture on small intestine transit and faecal water content, as well as the changing of intestinal microbial structures in rats were evaluated. Meanwhile, we also investigated several factors involved in signalling pathways that regulate function of ICC and expression of AQP3 to discuss the possible molecular mechanisms underlying the amelioration of constipation by the bacterial mixture.

\section{Materials and methods}

\section{Preparation of bacteria}

The bacterial preparation used in this study was a mixture of 3 strains, including B. breve DM8310, L. acidophilus DM8302, and L. casei DM8121. They were originally isolated from Chinese fermented food and deposited in the Culture Collection of Dalian Medical University (DMCC), Dalian, China. To identify these strains, $16 \mathrm{~S}$ rDNA of each strain was amplified by PCR and submitted to sequence. The partial sequences of $16 \mathrm{~S}$ rDNA of the 3 strains were deposited in NCBI GenBank under the accession number KP967559-KP967561. To prepare the bacterial mixture, the strains were firstly inoculated in the different culture media, BS agar (HB0394, Qingdao Hope Bio-Technology Co., Ltd., Qingdao City, China P.R.) medium for Bifidobacterium, and LBS agar (HB0385, Qingdao Hope Bio-Technology Co., Ltd.) medium for Lactobacillus. They were cultured under anaerobic conditions at $37^{\circ} \mathrm{C}$ for $24 \mathrm{~h}$. After that, single colonies were picked and inoculated in the corresponding liquid medium under the same conditions for $24 \mathrm{~h}$. The growth of bacteria was measured by optical density detection at a wavelength of $600($ OD600, OD600 $=0.5$ represents $10^{8}$ cells). Different volumes of bacteria were calculated and mixed according to the ratio of 1:1:1 (each strain $10^{9}$ cells) to form the bacterial consortium. After mixing, bacteria were harvested by centrifugation and resuspended in sterile phosphate buffered saline (PBS). The mixture was freshly made each day.

\section{Animal experiment}

For the animal experiment, 8 week old male Sprague Dawley (SD) rats ( $\mathrm{n}=21,210-270 \mathrm{~g}$ ) were obtained from the specific pathogen free animal centre of Dalian Medical University, Dalian, China. The animal experiments were performed under the approval of Dalian Medical University Institutional Animal Care and Use Committee in accordance with the laboratory's animal ethics guidelines (SYXK [Liao] 2014-0002). All rats were kept in a temperature-controlled room with ad libitum access to water and fasted for $24 \mathrm{~h}$ prior to all experiments. 
The experimental design is shown in Figure 1A. Except the control rats $(n=7)$, all the other 14 rats were intraperitoneally injected with loperamide $(5 \mathrm{mg} / \mathrm{kg}$, Sigma, St. Louis, MO, USA) twice a day (9:00 a.m. and 18:00 p.m.) from day 1 to day 5 to induce constipation. By day 6 , the 14 rats were randomly divided into a constipation group (Cons, $\mathrm{n}=7$ ) and a bacterial mixture intervention group (Mix, $n=7$ ). Rats in the Mix group were intragastricaly administrated $1 \times 10^{9} \mathrm{cfu} / \mathrm{kg}$ bacterial mixture suspended in $2 \mathrm{ml}$ PBS once a day for 7 days. Rats in the Control group and Cons group were administrated $2 \mathrm{ml}$ of PBS as vehicle. Mortality, body weight and faecal output was recorded daily. At day 13, all rats were executed via intraperitoneal anaesthesia (3\% chloral hydrate). The gastrointestinal tract was removed and emptied of contents. The transverse colon, blood, and faeces of rats were collected and frozen immediately at $-80{ }^{\circ} \mathrm{C}$ until detection.

\section{Small intestine transit}

Small intestine transit (SIT) is a test used and validated in several experimental studies on constipated rats (Han et al., 2016; Suo et al., 2014; Zhao et al., 2015). In our study, by day 12 , after administration of bacterial mixture, the rats were fasted for $24 \mathrm{~h}$. After that, they were intragastricaly administrated $1 \mathrm{ml}$ active carbon powder solution (3.0 g carbon powder suspended in $50.0 \mathrm{ml} 0.5 \%$ CMC-NA solution, Kermel Chemical Reagent Co., Ltd., Tianjin Shi, China P.R.). After $30 \mathrm{~min}$, the rats were executed and laparotomised to collect the small intestinal segments between stomach and ileocaecal junction. The active carbon was used as indicator. The distance from pylorus to ileocaecal junction was measured as the whole length of the small intestine. The distance from the pylorus to the frontier of carbon powder was measured as the migration distance of the active carbon. The active carbon propulsion rate was calculated by using the following formula: active carbon propulsion rate $(\%)=$ migration distance of active carbon/whole length of small intestine $\times 100 \%$.

\section{Detection of faecal water content}

Faecal samples were collected after sacrifice, and dried in an oven at $60{ }^{\circ} \mathrm{C}$ for $24 \mathrm{~h}$. Faecal water content (FWC) was calculated based on the following equation: [wet weights $(\mathrm{g})-$ dry weights $(\mathrm{g})] /$ wet weights $(\mathrm{g}) \times 100 \%$.

\section{Denaturing gradient gel electrophoresis profiling}

The meta-genomic DNA was extracted from the frozen faecal content of rats by the QIAamp DNA stool mini kit (Qiagen, Hilden, Germany). PCR was conducted using universal primers F338+GC clamp and R518 targeting the hyper-variable V3 region of $16 \mathrm{~S}$ rRNA gene. The resulting $16 \mathrm{~S}$ rDNA amplicons were analysed using the DCode system (Bio-Rad, Hercules, CA, USA) according to descriptions of Joossens et al. (2011). The digitised denaturing gradient gel electrophoresis (DGGE) images were analysed with Quantity One image analysis software (version 4.6.1; Bio-Rad). Similarities were displayed graphically as a dendrogram.

\section{S rDNA pyro-sequencing}

The universal primers (520F, 802R) were used to amplify the V3-V4 region of $16 \mathrm{~S}$ rDNA from metagenomic DNA in rat faeces. Primer sets were modified with Illumina adapter regions for sequencing on the IlluminaGAIIx platform (Illumina, San Diego, CA, USA). The reverse primers were modified with an 8-bp Hamming errorcorrecting barcode to distinguish among samples. The 50 $\mu \mathrm{l}$ PCR mixture contained the following components: 100 ng of DNA template, $5 \mu$ PCR buffer, $1 \mu \mathrm{l}$ dNTPs, 0.25 $\mu$ l HotStarTaq ${ }^{\oplus}$ Plus DNA Polymerase (Qiagen), and 2.5 pmol of each primer. The PCR program consisted of an initial step at $95^{\circ} \mathrm{C}$ for $5 \mathrm{~min} ; 30$ cycles of $94{ }^{\circ} \mathrm{C}$ for $45 \mathrm{~s}$, $55^{\circ} \mathrm{C}$ for $45 \mathrm{~s}$, and $72{ }^{\circ} \mathrm{C}$ for $60 \mathrm{~s}$; and a final extension at $72{ }^{\circ} \mathrm{C}$ for $8 \mathrm{~min}$. PCR products were checked by $1.5 \%(\mathrm{w} / \mathrm{v})$ agarose gel electrophoresis in $0.5 \mathrm{mg} / \mathrm{ml}$ ethidium bromide and purified with the Qiaquick gel extraction kit (Qiagen). Sequences of $16 \mathrm{~S}$ rDNA were detected by Illumina HiSeq 2000 platform (reconstructed cDNA sequence: $2 \times 150 \mathrm{bp}$ ). Ribosomal Database Project Classifier 2.8 was used for taxonomical assignment of all sequences at $50 \%$ confidence after the raw sequences were identified by their unique barcodes. Operational taxonomic units (OTUs) present in more than $50 \%$ the faecal samples were identified as core OTUs. Partial least squares discriminant analysis (PLSDA) of core OTUs was performed using Simca-P version 12 (Umetrics, Sartorius Stedim, Umeå, Sweden). The heat map was generated with Multi-Experiment Viewer $(\mathrm{MeV})$ software to visualise and cluster the microbial community into different groups. Community diversity was measured by the Shannon-Weiner biodiversity index (Shannon index). The analysis was conducted by Personal Biotechnology (Shanghai, China P.R.).

\section{Detection of neurotransmitter substance $\mathrm{P}, 5-\mathrm{HT}, \mathrm{Cl}^{-}$and $\mathrm{Ca}^{2+}$ levels in rat colon}

At the time of sacrifice, the transverse colons of rats were removed, washed with cold $\mathrm{PBS}$ and stored at $-80{ }^{\circ} \mathrm{C}$ for subsequent analysis. The concentrations of SP and 5-HT were assayed by ELISA (Omega Diagnostics, Alva, UK) according to the manufacture's instruction. The levels of $\mathrm{Cl}^{-}$ and $\mathrm{Ca}^{2+}$ were determined by commercially kits (Nanjing Jiancheng Bioengineering Institute, Nanjing, China P.R.) according to the manufacture's instruction. 


\section{Western blot of $\mathrm{C}$-kit and $\mathrm{AQP3}$ in rat colon}

The total protein was extracted from the transverse colon of rat. Equal amounts of proteins were fractioned with sodium dodecylsulfate polyacrylamide gel electrophoresis (SDS-PAGE) followed by electrophoretic transfer of proteins onto nitrocellulose membranes. The blots were probed with antibodies against $\mathrm{C}$-kit, AQP3, $\beta$-actin (Abcam, Cambridge, UK), and followed by incubation with secondary antibodies conjugated with horseradish peroxidase (HRP; Thermo Fisher, Waltham, MA, USA). The secondary antibodies were detected with a WesternBright ${ }^{\mathrm{mm}}$ ECL Western Blotting HRP Substrate kit and analysed with image lab software (BioRad, Hercules, CA, USA).

\section{qPCR detection of PKA, NK1, Tph1, Tph2, and Sert mRNA expressions in rat colon}

The transverse colon samples of rats were preserved in RNAlater solution. RNA was extracted by the RNeasy mini Kit (Qiagen). The complementary DNA (cDNA) was synthesised using the AffinityScript Multiple Temperature cDNA synthesis Kit (Stratagene, La Jolla, CA, USA) according to the supplier's protocol. cDNAs obtained by reverse transcription were used to determine mRNA expression levels of genes encoding PKA, NK1, Tph1, Tph2, and Sert by the specific primers as described previously (Ikarashi et al., 2011; Kunde et al., 2013; Yano et al., 2015). PCR reactions were run using the StepOnePlus Real-Time PCR System (Life Technologies, Carlsbad, CA, USA). The reaction mixture $(10 \mu \mathrm{l})$ comprised $4.5 \mu \mathrm{l}$ FastStart Universal SYBR Green Master (Roche, Mannheim, Germany), $0.5 \mu \mathrm{l}$ of each primer $30 \mu \mathrm{M}, 2.5 \mu \mathrm{l}$ of sterile distilled water, and 2 $\mu \mathrm{l}$ of DNA template $(100 \mathrm{ng} / \mu \mathrm{l})$. Each sample was repeated in triplicate. The mean $\mathrm{Ct}$ was determined from the three runs. Relative mRNA expression was expressed as $\Delta \mathrm{Ct}=$ $\mathrm{Ct}$ (target genes) - Ct (calibrator). $\beta$-actin housekeeping gene expression was used as the internal control. Then, relative mRNA expression was calculated as $\Delta \Delta \mathrm{Ct}=\Delta \mathrm{Ct}$ (treatment) $-\Delta \mathrm{Ct}$ (control). Finally, the relative gene expression levels were converted and expressed as fold difference $\left(=2^{-\Delta \Delta \mathrm{Ct}}\right)$.

\section{Statistical analysis}

Data were calculated by SPSS 19.0 statistical package (SPSS Inc., Chicago, IL, USA). Statistical analyses were performed using One-way ANOVA followed by Tukey's test with the assistance of GraphPad Prism Program (Version 5.01; GraphPad Software Inc., La Jolla, CA, USA). Values were considered significant at $P<0.05$.

\section{Results}

\section{Effects of the bacterial mixture on small intestine transit and faecal water content}

The experimental design is shown in Figure 1A. Loperamide induced a significant decrease in SIT in constipated rats by day 12 (from $57.73 \pm 1.24 \%$ to $33.43 \pm 1.88 \%, P=0.0002$; Figure
A

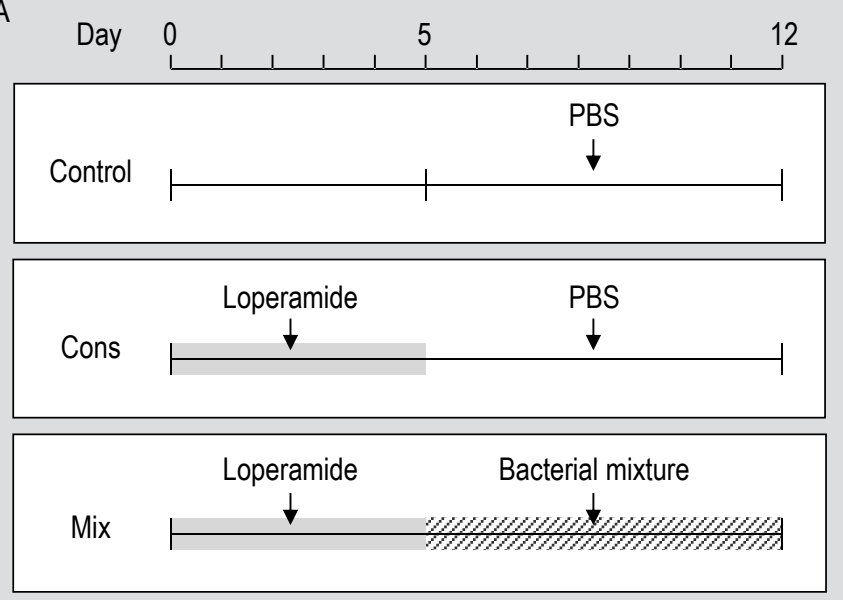

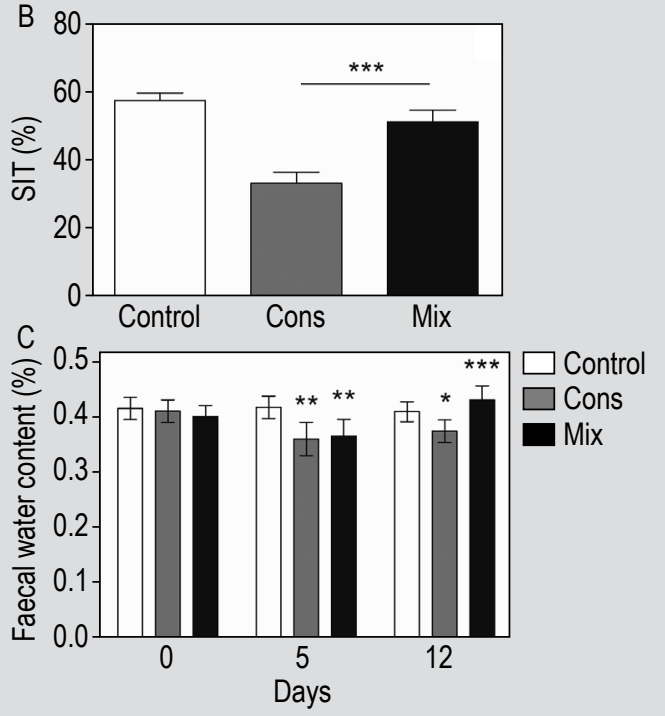

Figure 1. (A) Study design. Except for control rats ( $\mathrm{n}=7)$, rats were intraperitoneally injected with loperamide $(5 \mathrm{mg} / \mathrm{kg})$ twice each day from day 1 to day 5 to induce constipation. By day 6 , the loperamide-treated rats were divided into 2 groups, the constipation group (Cons, $n=7$ ), and the bacterial mixture intervention group (Mix, $n=7)$. Rats in the Mix group were intragastrically administered $1 \times 10^{9} \mathrm{cfu} / \mathrm{kg}$ bacterial mixture suspended in $2 \mathrm{ml}$ PBS once each day for seven days. Rats in the Control and Cons groups were given $2 \mathrm{ml}$ of PBS as vehicle. (B) Small intestine transit (SIT) of rats in each experimental group by day 12. (C) Faecal water content of rats in each group at days 5 and $12 .{ }^{*} P<0.05 ;{ }^{* *} P<0.01 ;{ }^{* * *} P<0.001$. All values were analysed for variance homogeneity test. 
1B). SIT significantly increased following intervention with the bacterial mixture compared with the constipation group $(54.43 \pm 2.03 \%, P=0.0025)$. The fresh faeces of each group were weighed and dried on days 1, 5, and 12. The water content of faeces was calculated (Figure 1C). On day 1 , the faecal water content was not significantly different between groups. After treatment with loperamide for 5 days, the faecal water content in the constipation group significantly decreased compared with the control group $(P=0.0015)$. After seven days of treatment with the bacterial mixture, an obvious improvement in faecal water content in the treatment group was observed versus the constipation group $(P=0.0006)$.

\section{Changes in intestinal microbiota post-intervention with the bacterial mixture}

We used PCR-DGGE to evaluate the possible effects of loperamide on the intestinal microbiota of rats and the changing of microbes post-intervention with the bacterial mixture. The DGGE fingerprints are shown in Figure 2A. The number of bands in each lane represents the diversity of the intestinal microbiota. Our data show that the abundance of intestinal microbes was significantly different between the control and constipation groups. DGGE fingerprints from the control group indicated an average of 37 bands per lane, whereas the average number of bands in the constipation group was 19 and the bacterial mixture treatment group showed an average of 30 bands. This suggested that loperamide induced a serious disruption of the intestinal microbiome that effectively modulated the intervention of the bacterial mixture. Cluster analysis showed that the constipation group was classified as a single category, and the control and the bacterial mixture treatment groups joined together, which suggested a recovery of the intestinal microbiota post-intervention with the bacterial mixture (Figure 2B).

To characterise the changing of gut microbiota systematically in rats post-intervention with the bacterial mixture, we performed metagenomic analysis of the V3-V4 region of $16 \mathrm{~S}$ rRNA gene sequences. The overall OTUs of intestinal bacteria differed between groups (Supplementary Figure S2A). Compared with the control group, the constipated rats had decreased OTU numbers that increased in the bacterial mixture-treated rats. Intervention with the bacterial mixture slightly, but not significantly, increased the Shannon index in rats, which suggested an improvement of bacterial diversity (Supplementary Figure $\mathrm{S} 2 \mathrm{~B})$. Administration of the bacterial mixture resulted in obvious modifications of the microbial structures of the rat intestine, which was proven by PLS-DA (Supplementary

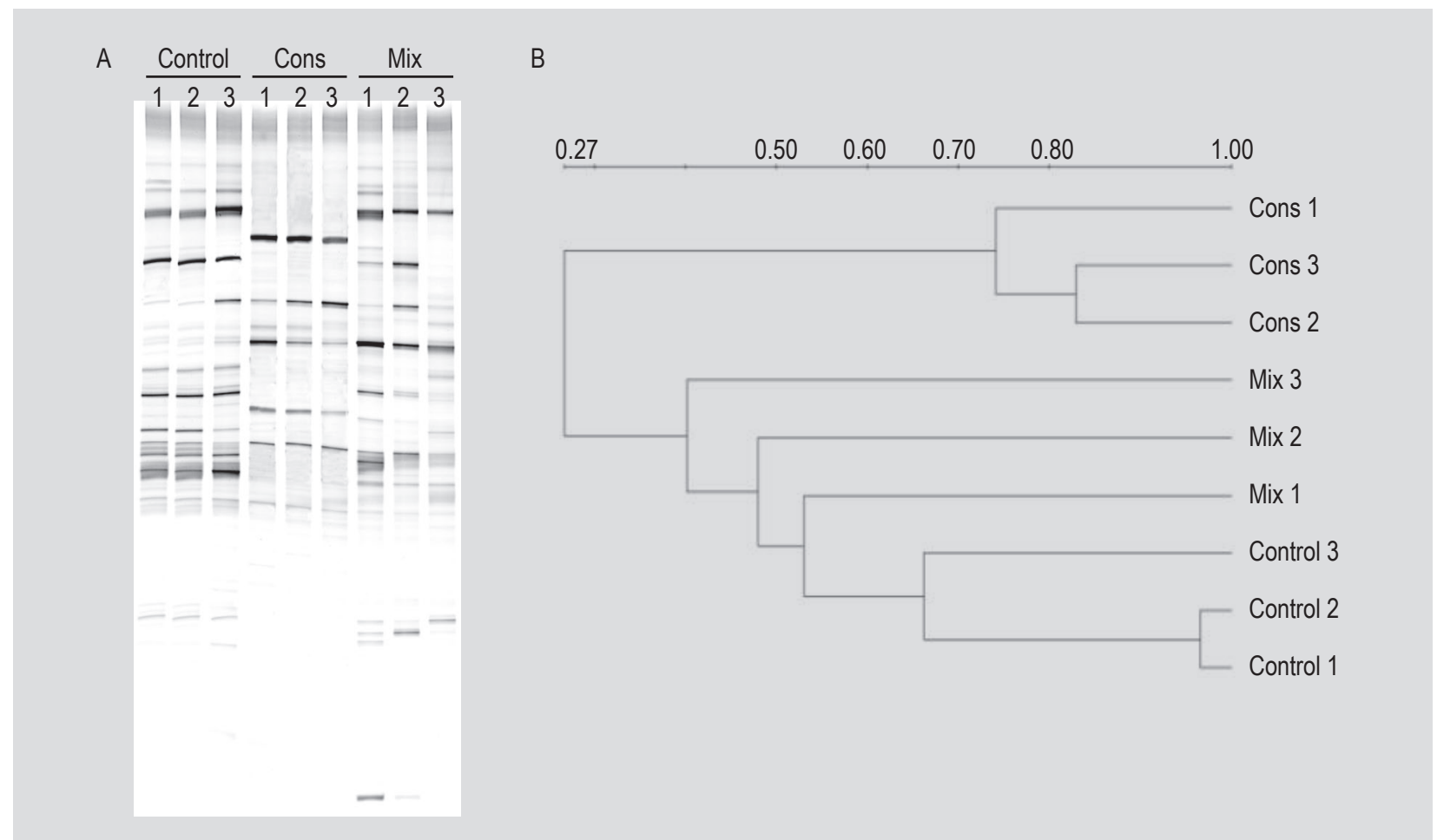

Figure 2. Preliminary evaluation of the intestinal microbiota in rats by PCR-denaturing gradient gel electrophoresis (DGGE). (A) DGGE profiles of the V3 region of $16 \mathrm{~S}$ rRNA gene amplicons derived from faecal DNA of rats in each group. PCR was performed using a F338-GC/R518 set of primers. Lanes indicate the microbial groups in faecal samples of the control group ( $n=3)$, constipated group $(n=3)$, and the bacterial mixture treated group $(n=3)$ taken at day 12. (B) Cluster analysis of the DGGE profiles. The dendrogram was constructed using the UPGMA method. 
Figure S2C). Changes were observed not only at the phylum level, but also at the levels of family and genus (Figure 3A). The MetStat analysis of gut microbes revealed that some specific microbial groups were inhibited or promoted by intervention of the bacterial mixture (Figure 3B). For example, the order of Clostridiales was largely reduced in constipated rats, but was increased in the bacterial mixturetreated rats; the genus of Lactobacillus, especially the species of Lactobacillus crispatus was elevated dramatically. Conversely, the groups of Bacteroidales, Erysipelotrichales, Prevotellaceae, and Enterococcaceae, which were elevated in constipated rats, were reduced by intervention of the bacterial mixture.

The metagenomic analysis LEfSe approach was applied to identify the key phylotypes responsible for the differences between groups. Firmicutes and Clostridia were most abundant in the control rats, while Bacteroidetes, Rikenellaceae, Burkholderiales, and Erysipelotrichales were most abundant in the constipated rats. Christensenellacea and Marvinbryantia were most abundant in the bacterial mixture-treated rats. These bacteria were the dominant phylotypes that contributed to the differences between the intestinal microbiota of different groups (Supplementary Figure S3).

\section{Effects of the bacterial mixture on the regulatory pathways of the interstitial cells of Cajal}

The expression of C-kit protein in the transverse colon of rats was detected by western blot (Figure 4A). Loperamide induced a significant reduction of $\mathrm{C}$-kit protein expression, while expression of $\mathrm{C}$-kit protein increased compared with the constipation group after the seven-day intervention with the bacterial mixture $(P=0.002)$. Loperamide also induced reduction of colon SP levels in constipated rats; however, these levels increased after intervention with the bacterial mixture in comparison with the constipation group (Figure $4 \mathrm{~B}, P=0.0056)$. As shown in Figure $4 \mathrm{C}$, the expression levels of NK1 gene mRNA significantly decreased in constipated rats following administration of loperamide $(P<0.001)$. However, there were no significant changes after treatment with the bacterial mixture relative to the constipation group, which suggests these may be related to its activity rather

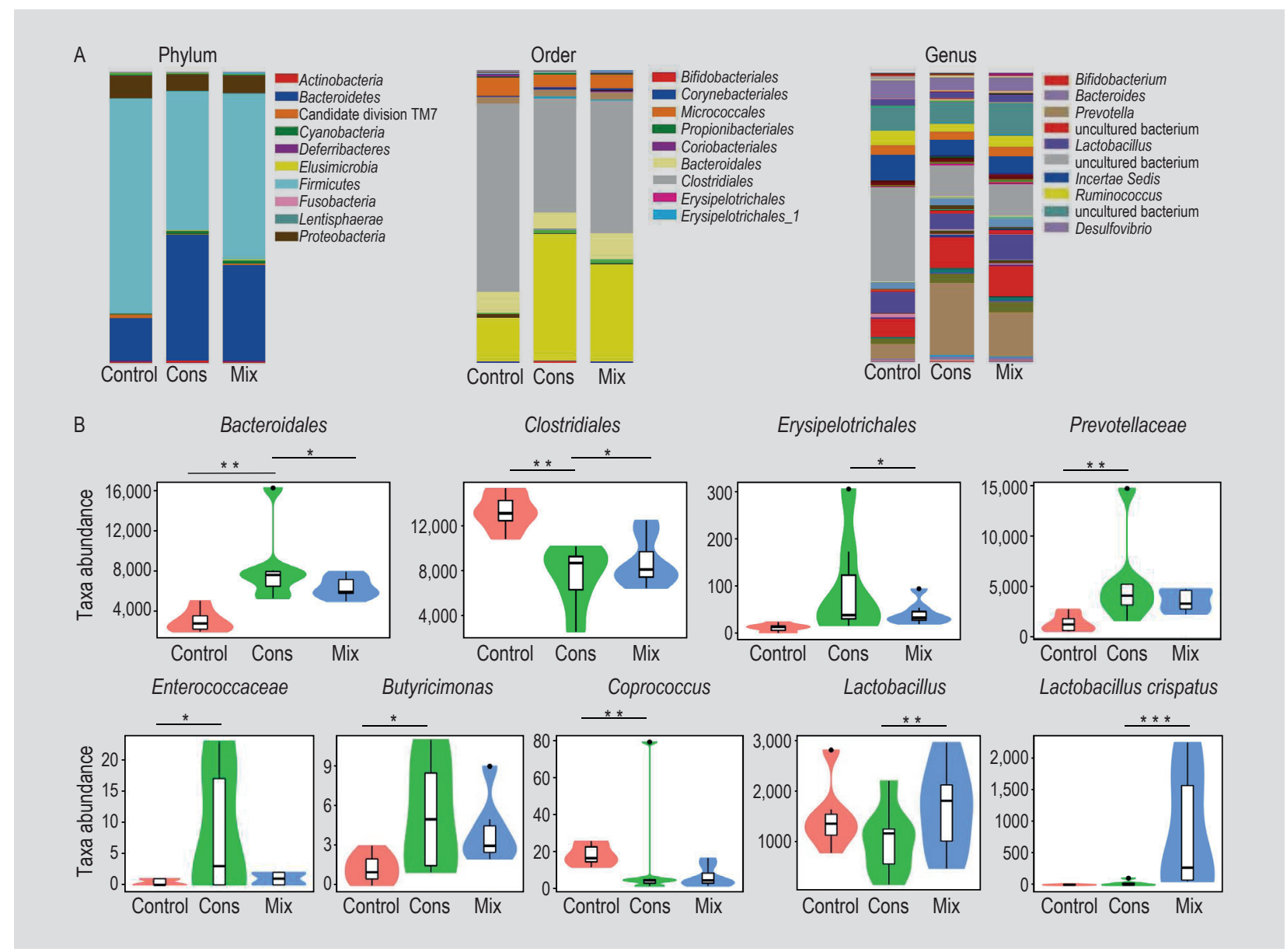

Figure 3. The bacterial mixture modulated the intestinal bacterial microbial composition in rats with loperamide-induced constipation. (A) The composition of bacterial population in different experimental groups at Phylum, Order, and Genus levels, respectively $(n=7)$. (B) Taxa abundance that are significantly different among groups identified using MetaStat method $(n=7)$. 

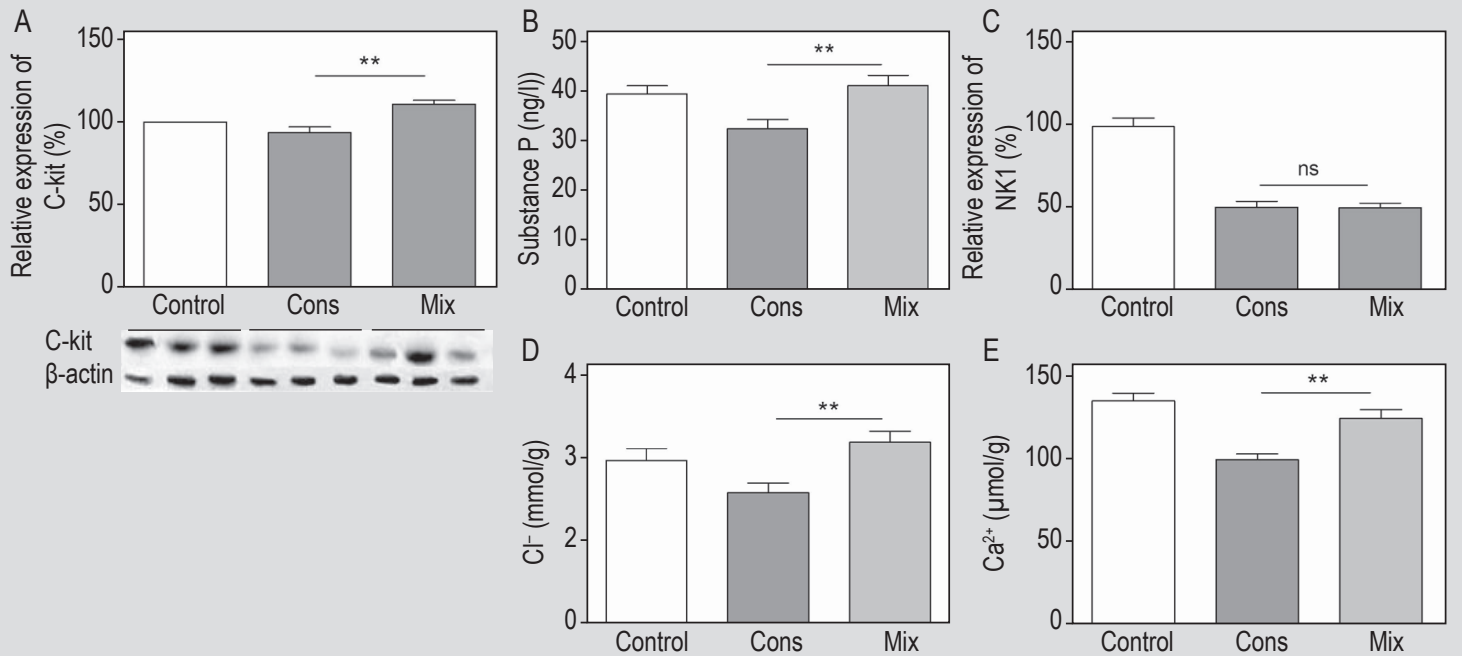

Figure 4. Effects of the bacterial mixture on signalling pathways of interstitial cells of Cajal in the colon of constipated rats. (A) Colonic C-kit protein expression of rats from different experimental groups detected by Western blot $(n=3)$. (B) Levels of substance $P$ in colonic tissues of rats from different experimental groups $(n=7)$. (C) Relative expression levels of neurokinin (NK)1 mRNA in rats of each group ( $n=7)$ detected by $q P C R$. (D) and (E) Levels of $\mathrm{Cl}^{-}$and $\mathrm{Ca}^{2+}$ in colonic tissues of rats from different experimental groups $(n=7)$. All values were analysed by variance homogeneity test. ${ }^{* *} P<0.01 ; n s=$ not significant.

than gene expression levels. The concentrations of $\mathrm{Cl}^{-}$and $\mathrm{Ca}^{2+}$ were significantly different between the constipation group and the bacterial mixture treatment group $(P=0.0038$, $P=0.0025)$ as shown in Figures 4D and 4E.

\section{Effects of the bacterial mixture on the expression levels of PKA and AQP3}

As shown in Figure 5A, the expression of PKA gene mRNA significantly decreased in constipated rats following administration of loperamide $(P<0.001)$, but increased after treatment with the bacterial mixture, although differences between the treatment group and the control group were not statistically significant $(P>0.05)$. The expression of AQP3 protein in the transverse colon of rats in different groups is shown in Figure 5B. After 7 days of bacterial treatment, the expression of AQP3 protein significantly increased in the treatment group compared with the constipation group $(P=0.0155)$.
A

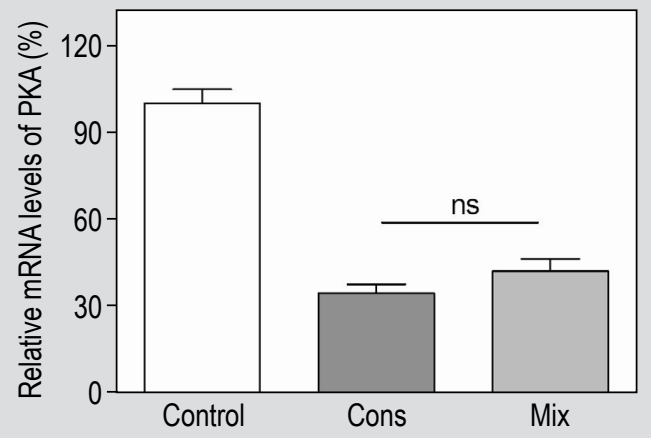

B

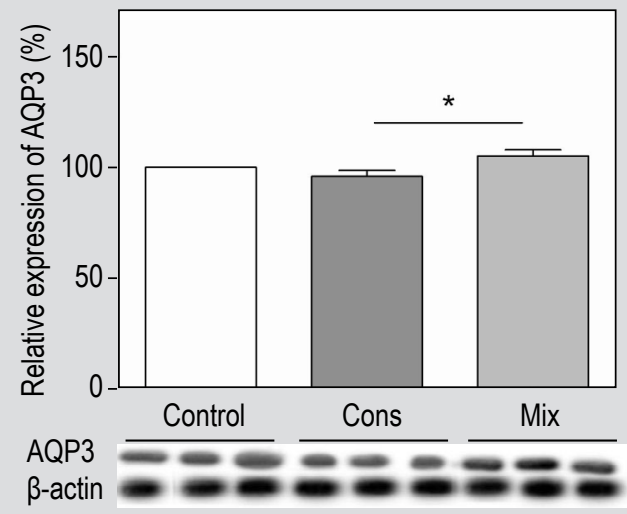

Figure 5. Effects of the bacterial mixture on protein kinase $A(P K A)$ and aquaporin (AQP)3 in the colon of constipated rats. (A) Relative expression levels of PKA mRNA in rats of each group ( $n=7$ ) detected by qPCR. (B) Colonic AQP3 protein expression of rats from different experimental groups detected by western blot $(n=3)$. All values were analysed by variance homogeneity test. ${ }^{*} P<0.05 ;$ ns $=$ not significant. 


\section{Effects of the bacterial mixture on 5-HT biosynthesis in the rat colon}

To test whether the improvement of SIT and faecal water content of constipated rats by the bacterial mixture were correlated with changing levels of intestinal 5-HT, we detected 5-HT expression using an ELISA. Results in Figure $6 \mathrm{~A}$ show that the constipated rats exhibited lower levels of 5-HT in their colonic tissues, and these levels increased following intervention with the bacterial mixture, although the elevation was not statistically significant $(P>0.05$ by one-way ANOVA followed by Tukey's test). We further evaluated the mRNA expression levels of Tph1 and Tph2, genes that control the biosynthesis of 5-HT in ECs, and Sert, which encodes the 5-HT transporter gene. Results shown in Figures $6 \mathrm{~B}$ and $6 \mathrm{C}$ suggest that treatment with the bacterial mixture up-regulates the biosynthesis of 5-HT in the rat colon (all $P<0.05$ ), but does not affect 5 -HT transport (Figure 6D).

\section{Discussion and conclusions}

Many studies have investigated the effects of different bacterial strains as potential probiotics using constipated animal models (Li et al., 2015; Wang et al., 2017; Zhao et al., 2015). Clinical trials testing the application of probiotics show promising results and support intervention with probiotics as a feasible way to ameliorate constipation (Kim et al., 2015; Wojtyniak et al., 2017). In our previous study, a bacterial mixture of four different probiotic strains was successfully applied to the treatment of the intestinal dysbiosis (Wang et al., 2017). Wang et al. (2014) found that a mixture of three different bacterial strains was superior in restoring intestinal barrier function and reducing inflammation compared with individual probiotic strains. However, to our knowledge, few of these studies systematically evaluated changes in the intestinal microbiota post-intervention with potential probiotics, although previous studies suggested a close association between constipation and altered abundance of certain taxa of the intestinal microbiome. Kim et al. (2015) found that Bifidobacteria and Bacteroides species were significantly less abundant in the faeces of patients with FC compared with that of healthy controls. Additionally, no significant differences were observed regarding the proportion of Lactobacillus, Escherichia coli and Clostridium species.

In our study, the constipation group showed an increased abundance in faecal microbiota of bacterial orders, such as Bacteroidales and Erysipelotrichales, families, such as Bacteroideaceae, Prevetollaceae, and the genera of Bacteroidetes and Parabacteroidetes. In contrast, the
A

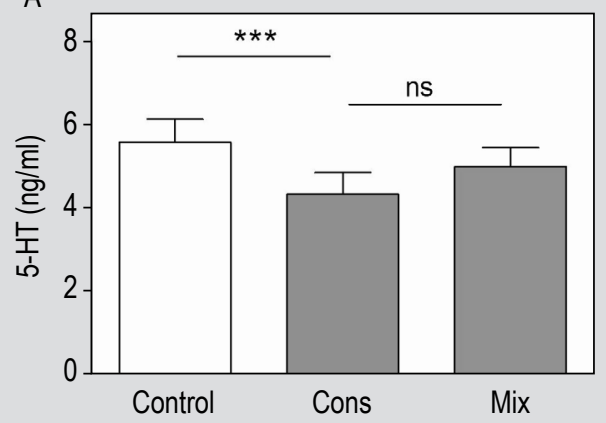

C

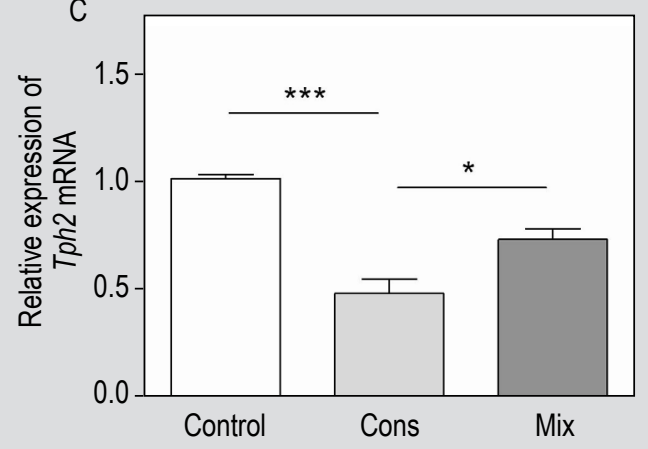

B

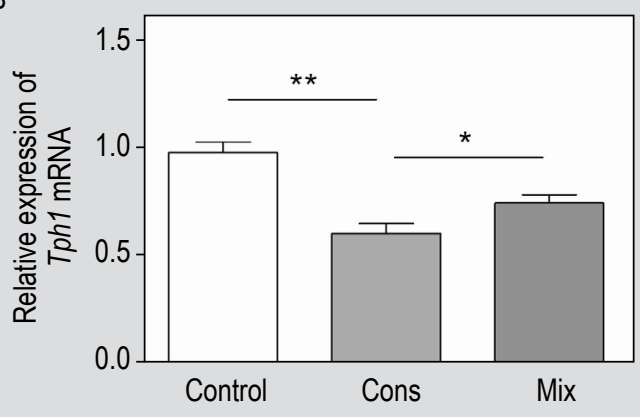

D

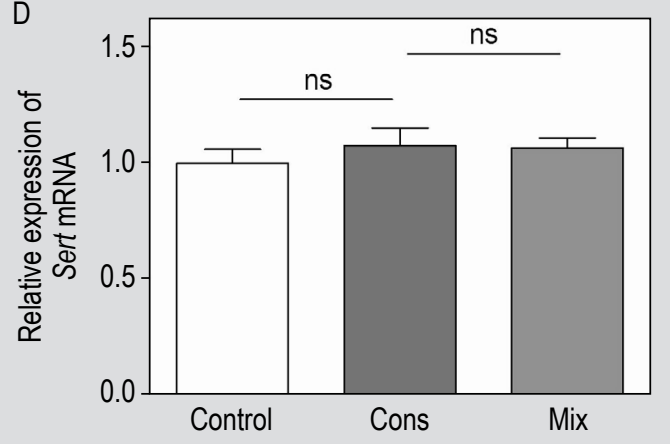

Figure 6. Effects of the bacterial mixture on the biosynthesis of 5-HT in the colon of constipated rats. (A) Levels of 5-HT in colonic tissues of rats from different experimental groups ( $n=7)$. Relative expression levels of (B) tph1, (C) tph2, and (D) sert mRNA in rats of each group $(n=7)$ detected by $\mathrm{qPCR}$. All values were analysed by variance homogeneity test. ${ }^{*} P<0.05,{ }^{* *} P<0.01,{ }^{* * *} P<0.001$, ns $=$ not significant. 
order of Clostridiales, family of Lactobacillaceae, and the genus Lactobacillus decreased in the stool of these rats. Compared with the constipation group, the abundance of genera of Bacteroides, Prevotella and Bifidobacterium decreased in the bacterial mixture-treated group, while the abundance of genus Lactobacillus increased. These results are partially consistent with previous studies that analysed the faecal microbiota in patients with FC. Lactobacillus had been shown to be significantly less abundant in adult patients with constipation (Chassard et al., 2012; Khalif et al., 2005). Previous studies also showed that butyrateproducing genera, such as Coprococcus, Roseburia, and Faecalibacterium, tended to be increased in constipated patients (Pryde et al., 2002; Sokol et al., 2008). These data were consistent with our results in that the genera of Coprococcus, Butyricimonas were increased in constipated rats, while being inhibited by intervention with the bacterial mixture. However, our data demonstrated inconsistent results compared with other previous studies. For example, the abundance of Clostridiales significantly decreased in constipated rats, but was increased post-intervention with the bacterial mixture. In contrast, the abundance of Bifidobacterium was not significantly different among groups. These results differ from a previous paediatric study, which indicated that Clostridia and Bifidobacteria were significantly increased in the faeces of constipated children. Because the Clostridium species isolated from constipated children were found to be different from those of healthy controls (Zoppi et al., 1998), we concluded that our results might represent a typical changing of the gut microbiota in rats with loperamide-induced constipation, but not in murine models or constipated children.

Manipulation of the intestinal microbiota using a bacterial mixture may contribute to the improvement of SIT and faecal water content in constipated rats. Many studies have shown an interaction between the intestinal microbiome and the enteric nervous system (ENS) (Hyland and Cryan, 2016). The ENS regulates gastrointestinal physiology and function, in part through secretion of neuropeptides, such as SP (Lakhan and Kirchgessner, 2010). To clarify the mechanisms underlying the ameliorative effects of the bacterial mixture on loperamide-induced constipation, we detected the major signalling pathway involved in the regulation of ICC-mediated gut motility. ICC played a significant physiological role in intestinal motility (Sanders and Ward, 2007). ICC transduced inputs from enteric motor neurons and generated intrinsic electrical rhythmicity, which was a minor component of the tunica muscularis of the GI tract. The rhythmic potential was mainly composed of $\mathrm{Ca}^{2+}$ and $\mathrm{Cl}^{-}$channel participation. Many ICC expressed C-kit, which is a membrane receptor with tyrosine kinase activity (Takaki, 2003). Expression of C-kit has been shown in rat models with slow transit constipation (Tong et al., 2005). In our study, the expression of C-kit protein was down-regulated in constipated rats, but was up-regulated by intervention with the bacterial mixture, which suggested that the C-kit signalling pathway may be essential in ICC for the regulation of gut motility. The tachykinin receptor (NK1, NK2 and NK3) was detected in all tissues studied with expression levels differing by up to 2,500-fold between tissues (Kunde et al., 2013). Among these, NK1 was identified in ICC. Evidence was provided that SP may modulate intestinal motility by nonselective action on cation channels on ICC via the release of intracellular $\mathrm{Ca}^{2+}$ induced by tachykinin NK1 receptor stimulation (Jun et al., 2004; Watson and Downes, 1983). Our study found that levels of $\mathrm{SP}, \mathrm{Ca}^{2+}$, and $\mathrm{Cl}^{-}$in colonic tissues of rats were up-regulated by intervention with the bacterial mixture. Although we did not directly detect these indicators of the small intestine, changes in colonic peristalsis may indirectly contribute to the improvement of small intestinal transit. However, detection of the mRNA expression of NK1 showed no obvious changing, either in the constipated rats or in the bacterial mixture-treated rats, which suggested that the kinase activity but not expression level of these proteins was responsible for the activation of ICC.

Faecal water content was another important condition affecting the status of constipation. The water channels AQPs play important roles in the water transport system in human body. AQP3 is predominantly expressed in mucosal epithelial cells of colon; thus, it is believed to play a pivotal role in controlling the water transport of the colon. However, to date, little is known about the relationship between AQP and constipation. AQP3 in the colon of rat models with slow transit constipation was down-regulated (Zhi and Yuan, 2011), which was consistent with our results (Figure 6). Previous studies demonstrated expression of AQP3 protein in human amniotic epithelial cells (hAECs) was regulated by the cAMP-PKA-CREB signalling pathway (Hua et al., 2015). We found that intervention with the bacterial mixture resulted in elevation of faecal water contents in the rat intestine. Enhanced kinase activity of PKA may contribute to the up-regulation of AQP3, but not the mRNA expression of the gene encoding this protein kinase, given that the mRNA expression levels of the PKA gene in rat colonic tissues did not change significantly.

However, it was still unclear how changing of the intestinal microbiome affected gut motility and faecal water content. 5-HT was found to up-regulate both SP/NK1 receptor pathways and the expression of AQP3 (Kon et al., 2015; Utsumi et al., 2016). Therefore, we assumed that 5-HT secretion levels from intestinal ECs of rats may be affected by the bacterial mixture because intestinal biosynthesis of $5-\mathrm{HT}$ is regulated by indigenous microbial groups, especially spore-forming bacteria, such as Clostridia species (Yano et al., 2015). Antibiotic-induced depletion of mice microbiota led to changes in host 5-HT biosynthesis and intestinal motility (Ge et al., 2017). Our results of 16S rDNA sequencing did find promotion of intestinal Clostridiales in 
constipated rats by intervention with the bacterial mixture. Except for Clostridiales, microbial groups producing shortchain fatty acids were found to stimulate ECs to promote colonic 5-HT production (Reigstad et al., 2015). We also found some microbial groups, such as Lactobacillus, were promoted by intervention with the bacterial mixture. However, 16S rDNA sequencing has been demonstrated to have a limited taxonomical and functional resolution. Bacterial strain identification was an issue that remained unsatisfactory with the current approach. Only a narrow portion of all the strains in the colon can be recognised because of general unculturability. This might have some impact on the intestinal flora of the detection rate in our experiments. In addition to biosynthesis, the transporter for 5-HT was found to be inhibited by activation of toll-like receptor 2, which suggests a role for gut microbes in 5-HT transport (Latorre et al., 2016). Our detection of 5-HT levels in rat colon showed that treatment with loperamide resulted in an obvious reduction of 5-HT, but this was reversed by intervention with the bacterial mixture. Although the elevation of 5-HT levels was not statistically significant, we did find an increase in the expression of 5-HT biosynthesis genes (Tph1 and Tph2), but not Sert, which encodes the transporter for 5-HT.

In conclusion, intervention with the bacterial mixture ameliorated loperamide-induced constipation in rats. The up-regulation of C-kit/SP signalling pathways in ICC and AQP3 significantly contributed to the improvement of SIT and faecal water content. These changes were closely associated with the manipulation of intestinal dysbiosis in the constipated rats by the bacterial mixture. Our results further revealed the important role of the intestinal microbiota in affecting intestinal motility through regulation of 5-HT biosynthesis. This suggests that the disrupted microbiome in patients can be a potential therapeutic target for the improvement of constipation through intervention with probiotics.

\section{Supplementary material}

Supplementary material can be found online at https://doi. org/10.3920/BM2017.0062.

Figure S1. The intestinal microbial composition in individual animals between different groups. Figure S2. The bacterial mixture modulated intestinal bacterial microbial composition in rats with loperamide-induced constipation.

Figure S3. LEfSe analysis of bacterial microbiota among experimental animal groups.

\section{Acknowledgements}

We thank Joel Anderson, PhD, from Liwen Bianji, Edanz Group China (www.liwenbianji.cn/ac), for editing the English text of this manuscript. This work was supported by the Nature Science Foundation of China (81302282), the Nature Science Foundation of Liaoning Province, China (2015020262), the China Postdoctoral Science Foundation (2016M601317), and the Research foundation from the Department of Education, Liaoning Province, China (L2016003).

\section{Conflict of interest}

The authors declare that they have no conflict of interest.

\section{References}

Chassard, C., Dapoigny, M., Scott, K.P., Crouzet, L., Del'homme, C., Marquet, P., Martin, J.C., Pickering, G., Ardid, D., Eschalier, A., Dubray, C., Flint, H.J. and Bernalier-Donadille, A., 2012. Functional dysbiosis within the gut microbiota of patients with constipated-irritable bowel syndrome. Alimentary Pharmacology and Therapeutics 35: 828-838.

Daniel, E.E., 2001. Physiology and pathophysiology of the interstitial cell of Cajal: from bench to bedside. III. Interaction of interstitial cells of Cajal with neuromediators: an interim assessment. American Journal of Physiology, Gastrointestinal and Liver Physiology 281: G1329-1332.

Ge, X., Ding, C., Zhao, W., Xu, L., Tian, H., Gong, J., Zhu, M., Li, J. and Li, N., 2017. Antibiotics-induced depletion of mice microbiota induces changes in host serotonin biosynthesis and intestinal motility. Journal of Translational Medicine 15: 13.

Han, S.H., Hong, K.B., Kim, E.Y., Ahn, S.H. and Suh, H.J., 2016. Effect of dual-type oligosaccharides on constipation in loperamide-treated rats. Nutrition Research and Practice 10: 583-589.

Hua, Y., Ding, S., Zhang, W., Zhou, Q., Ye, W., Chen, M. and Zhu, X., 2015. Expression of AQP3 protein in hAECs is regulated by Camp-PKA-CREB signalling pathway. Frontiers in Bioscience 20: 1047-1055.

Huizinga, J.D., Thuneberg, L., Kluppel, M., Malysz, J., Mikkelsen, H.B. and Bernstein, A., 1995. W/kit gene required for interstitial cells of Cajal and for intestinal pacemaker activity. Nature 373: 347-349.

Hyland, N.P. and Cryan, J.F., 2016. Microbe-host interactions: Influence of the gut microbiota on the enteric nervous system. Developmental Biology 417: 182-187.

Ikarashi, N., Mochiduki, T., Takasaki, A., Ushiki, T., Baba, K., Ishii, M., Kudo, T., Ito, K., Toda, T., Ochiai, W. and Sugiyama, K., 2011. A mechanism by which the osmotic laxative magnesium sulphate increases the intestinal aquaporin 3 expression in HT-29 cells. Life Sciences 88: 194-200.

Jeong, D., Kim, D.H., Kang, I.B., Kim, H., Kim, H.S., Seo, K.H. and Song, K.Y., 2017. Modulation of gut microbiota and increase in fecal water content in mice induced by administration of Lactobacillus kefiranofaciens DN1. Food and Function 8: 680-686. 
Joossens, M., Huys, G., Cnockaert, M., De Preter, V., Verbeke, K., Rutgeerts, P., Vandamme, P. and Vermeire, S., 2011. Dysbiosis of the faecal microbiota in patients with Crohn's disease and their unaffected relatives. Gut 60: 631-637.

Jun, J.Y., Choi, S., Yeum, C.H., Chang, I.Y., You, H.J., Park, C.K., Kim, M.Y., Kong, I.D., Kim, M.J., Lee, K.P., So, I. and Kim, K.W., 2004. Substance P induces inward current and regulates pacemaker currents through tachykinin NK1 receptor in cultured interstitial cells of Cajal of murine small intestine. European Journal of Pharmacology 495: 35-42.

Khalif, I.L., Quigley, E.M., Konovitch, E.A. and Maximova, I.D., 2005. Alterations in the colonic flora and intestinal permeability and evidence of immune activation in chronic constipation. Digestive and Liver Disease: Official Journal of the Italian Society of Gastroenterology and the Italian Association for the Study of the Liver 37: 838-849.

Kim, S.E., Choi, S.C., Park, K.S., Park, M.I., Shin, J.E., Lee, T.H., Jung, K.W., Koo, H.S. and Myung, S.J., 2015. Change of fecal flora and effectiveness of the short-term VSL\#3 probiotic treatment in patients with functional constipation. Journal of Neurogastroenterology and Motility 21: 111-120.

King, L.S., Kozono, D. and Agre, P., 2004. From structure to disease: the evolving tale of aquaporin biology. Nature reviews. Molecular Cell Biology 5: 687-698.

Koh, S.D., Sanders, K.M. and Ward, S.M., 1998. Spontaneous electrical rhythmicity in cultured interstitial cells of cajal from the murine small intestine. Journal of Physiology 513(1): 203-213.

Kon, R., Ikarashi, N., Hayakawa, A., Haga, Y., Fueki, A., Kusunoki, Y., Tajima, M., Ochiai, W., Machida, Y. and Sugiyama, K., 2015. Morphine-induced constipation develops with increased aquaporin-3 expression in the colon via increased serotonin secretion. Toxicological Sciences 145: 337-347.

Kunde, D.A., Crawford, A. and Geraghty, D.P., 2013. Tachykinin (NK1, NK2 and NK3) receptor, transient receptor potential vanilloid 1 (TRPV1) and early transcription factor, cFOS, mRNA expression in rat tissues following systemic capsaicin treatment. Regulatory Peptides 183: 35-41.

Lakhan, S.E. and Kirchgessner, A., 2010. Neuroinflammation in inflammatory bowel disease. Journal of Neuroinflammation 7: 37.

Latorre, E., Layunta, E., Grasa, L., Castro, M., Pardo, J., Gomollon, F., Alcalde, A.I. and Mesonero, J.E., 2016. Intestinal serotonin transporter inhibition by toll-like receptor 2 activation. A feedback modulation. PLoS ONE 11: e0169303.

Li, C., Nie, S.P., Zhu, K.X., Xiong, T., Li, C., Gong, J. and Xie, M.Y., 2015. Effect of Lactobacillus plantarum NCU116 on loperamideinduced constipation in mice. International Journal of Food Sciences and Nutrition 66: 533-538.

Pryde, S.E., Duncan, S.H., Hold, G.L., Stewart, C.S. and Flint, H.J., 2002. The microbiology of butyrate formation in the human colon. FEMS Microbiology Letters 217: 133-139.

Reigstad, C.S., Salmonson, C.E., Rainey $3^{\text {rd }, ~ J . F ., ~ S z u r s z e w s k i, ~ J . H ., ~}$ Linden, D.R., Sonnenburg, J.L., Farrugia, G. and Kashyap, P.C., 2015. Gut microbes promote colonic serotonin production through an effect of short-chain fatty acids on enterochromaffin cells. FASEB Journal 29: 1395-1403.
Sanders, K.M., 1996. A case for interstitial cells of Cajal as pacemakers and mediators of neurotransmission in the gastrointestinal tract. Gastroenterology 111: 492-515.

Sanders, K.M. and Ward, S.M., 2007. Kit mutants and gastrointestinal physiology. Journal of Physiology 578: 33-42.

Sokol, H., Pigneur, B., Watterlot, L., Lakhdari, O., Bermudez-Humaran, L.G., Gratadoux, J.J., Blugeon, S., Bridonneau, C., Furet, J.P., Corthier, G., Grangette, C., Vasquez, N., Pochart, P., Trugnan, G., Thomas, G., Blottiere, H.M., Dore, J., Marteau, P., Seksik, P. and Langella, P., 2008. Faecalibacterium prausnitzii is an anti-inflammatory commensal bacterium identified by gut microbiota analysis of Crohn disease patients. Proceedings of the National Academy of Sciences of the USA 105: 16731-16736.

Suares, N.C. and Ford, A.C., 2011. Prevalence of, and risk factors for, chronic idiopathic constipation in the community: systematic review and meta-analysis. American Journal of Gastroenterology 106: 1582-1591.

Suo, H., Zhao, X., Qian, Y., Li, G., Liu, Z., Xie, J. and Li, J., 2014. Therapeutic effect of activated carbon-induced constipation mice with Lactobacillus fermentum Suo on treatment. International Journal of Molecular Sciences 15: 21875-21895.

Takaki, M., 2003. Gut pacemaker cells: the interstitial cells of Cajal (ICC). Journal of Smooth Muscle Research (Nihon Heikatsukin Gakkai kikanshi) 39: 137-161.

Tong, W.D., Liu, B.H., Zhang, L.Y., Xiong, R.P., Liu, P. and Zhang, S.B., 2005. Expression of c-kit messenger ribonucleic acid and c-kit protein in sigmoid colon of patients with slow transit constipation. International Journal of Colorectal Disease 20: 363-367.

Utsumi, D., Matsumoto, K., Amagase, K., Horie, S. and Kato, S., 2016. 5-HT3 receptors promote colonic inflammation via activation of substance P/neurokinin-1 receptors in dextran sulphate sodiuminduced murine colitis. British Journal of Pharmacology 173: 18351849.

Wang, H., Gong, J., Wang, W., Long, Y., Fu, X., Fu, Y., Qian, W. and Hou, X., 2014. Are there any different effects of Bifidobacterium, Lactobacillus and Streptococcus on intestinal sensation, barrier function and intestinal immunity in PI-IBS mouse model? PLoS ONE 9: e90153.

Wang, L., Hu, L., Xu, Q., Yin, B., Fang, D., Wang, G., Zhao, J., Zhang, H. and Chen, W., 2017. Bifidobacterium adolescentis exerts strainspecific effects on constipation induced by loperamide in BALB/c mice. International Journal of Molecular Sciences 18: 318.

Watson, S.P. and Downes, C.P., 1983. Substance P induced hydrolysis of inositol phospholipids in guinea-pig ileum and rat hypothalamus. European Journal of Pharmacology 93: 245-253.

Wojtyniak, K., Horvath, A., Dziechciarz, P. and Szajewska, H., 2017. Lactobacillus casei rhamnosus Lcr35 in the management of functional constipation in children: a randomized trial. Journal of Pediatrics 184: 101-105.

Yano, J.M., Yu, K., Donaldson, G.P., Shastri, G.G., Ann, P., Ma, L., Nagler, C.R., Ismagilov, R.F., Mazmanian, S.K. and Hsiao, E.Y., 2015. Indigenous bacteria from the gut microbiota regulate host serotonin biosynthesis. Cell 161: 264-276.

Yu, T., Zheng, Y.P., Tan, J.C., Xiong, W.J., Wang, Y. and Lin, L., 2017. Effects of prebiotics and synbiotics on functional constipation. American Journal of the Medical Sciences 353: 282-292. 


\section{Y. Deng et al.}

Zhao, X., Suo, H.Y., Qian, Y., Li, G.J., Liu, Z.H. and Li, J., 2015. Therapeutic effects of Lactobacillus casei Qian treatment in activated carbon-induced constipated mice. Molecular Medicine Reports 12: 3191-3199.

Zhi, H. and Yuan, W.T., 2011. Expression of aquaporin 3, 4, and 8 in colonic mucosa of rat models with slow transit constipation. Chinese Journal of Gastrointestinal Surgery (Zhonghua Wei Chang Wai Ke Za Zhi) 14: 459-461.
Zhu, L., Liu, W., Alkhouri, R., Baker, R.D., Bard, J.E., Quigley, E.M. and Baker, S.S., 2014. Structural changes in the gut microbiome of constipated patients. Physiological Genomics 46: 679-686.

Zhu, S., Ran, J., Yang, B. and Mei, Z., 2017. Aquaporins in digestive system. Advances in Experimental Medicine and Biology 969: 123-130.

Zoppi, G., Cinquetti, M., Luciano, A., Benini, A., Muner, A. and Bertazzoni Minelli, E., 1998. The intestinal ecosystem in chronic functional constipation. Acta Paediatrica 87: 836-841. 